\title{
Cocaine Evokes Projection-Specific Synaptic Plasticity of Lateral Habenula Neurons
}

\author{
Matthieu Maroteaux $x^{1,2,3}$ and Manuel Mameli ${ }^{1,2,3}$ \\ ${ }^{1}$ Institut du Fer à Moulin, 75005 Paris, France, ${ }^{2}$ INSERM, UMR-S839, 75005 Paris, France, and ${ }^{3}$ Université Pierre et Marie Curie, 75005 Paris, France
}

Addictive drugs share the ability to increase dopamine (DA) levels and trigger synaptic adaptations in the mesocorticolimbic system, two cellular processes engaged in the early stages of drug seeking. Neurons located in the lateral habenula (LHb) modulate the activity of DA neurons and DA release, and adaptively tune goal-directed behaviors. Whether synaptic modifications in LHb neurons occur upon drug exposure remains, however, unknown. Here, we assessed the influence of cocaine experience on excitatory transmission onto subsets of LHb neurons using a combination of retrograde tracing and ex vivo patch-clamp recordings in mice. Recent evidence demonstrates that AMPA receptors lacking the GluA2 subunit mediate glutamatergic transmission in LHb neurons. We find that cocaine selectively potentiates AMPA receptor-mediated EPSCs in LHb neurons that send axons to the rostromedial tegmental nucleus, a GABAergic structure that modulates the activity of midbrain DA neurons. Cocaine induces a postsynaptic accumulation of AMPA receptors without modifying their subunit composition or single-channel conductance. As a consequence, a protocol pairing presynaptic glutamate release with somatic hyperpolarization, to increase the efficiency of GluA2-lacking AMPA receptors, elicited a long-term potentiation in neurons only from cocaine-treated mice. This suggests that cocaine resets the rules for the induction of synaptic long-term plasticity in the LHb. Our study unravels an early, projection-specific, cocaine-evoked synaptic potentiation in the LHb that may represent a permissive step for the functional reorganization of the mesolimbic system after drug exposure.

\section{Introduction}

Midbrain dopamine (DA) neurons are central in guiding motivated behaviors as well as drug seeking (Wise, 2004). Addictive substances with different properties modulate DA neuron activity and DA release from the ventral tegmental area (VTA) (Lüscher and Malenka, 2011). Recent evidence indicates that the lateral habenula $(\mathrm{LHb}$ - a hub-like region anatomically and functionally connected to midbrain structures-bidirectionally modulates the activity of DA neurons (Matsumoto and Hikosaka, 2007). LHb neurons provide DA neurons with the signal necessary to respond to salient rewarding and aversive stimuli (Hikosaka, 2010). The LHb neurons are mostly glutamatergic, and contain GluA2-lacking AMPA receptors (AMPARs) (Li et al., 2011). They project to DA neurons through two distinct pathways (Balcita-Pedicino et al., 2011). The direct projection that targets the VTA has been recently implicated in the etiology of mood disorders (Li et al., 2011). The indirect projection tunes DA neuron activity through a GABAergic relay named rostromedial tegmental nucleus (RMTg) or tail VTA (Jhou et al., 2009;

\footnotetext{
Received May 18, 2012; revised July 19, 2012; accepted July 23, 2012.

Author contributions: M. Mameli designed research; M. Maroteaux and M. Mameli performed research; M. Maroteaux and M. Mameli analyzed data; M. Maroteaux and M. Mameli wrote the paper.

This work was supported by The Paris Neuroscience School (ENP), INSERM ATIP-AVENIR, and the City of Paris. We thank C. Bellone, M. Brown, M. Carta, F. J. Meye, C. Lüscher, C. F. Valenzuela, J. C. Poncer, and the whole Poncer-Levi Laboratory for helpful discussions and comments. We thank C. F. Valenzuela for assistance with English language editing, and N. Gervasi and L. Gonnard for image acquisition.

The authors declare no competing financial interests.

Correspondence should be addressed to Dr. Manuel Mameli, Institut du Fer a Moulin, UMR-S 839 INSERM/UPMC, Batiment INSERM, 8-10 Rue Fosses Saint Marcel, 75005 Paris. E-mail: manuel.mameli@inserm.fr.

DOI:10.1523/JNEUROSCI.2405-12.2012

Copyright $\odot 2012$ the authors $\quad 0270-6474 / 12 / 3212641-06 \$ 15.00 / 0$
}

Kaufling et al., 2009). Addictive drugs have been shown to alter the physiology and the expression of immediate early genes, not only in VTA DA neurons, but also in putative RMTg and LHb neurons (Brown et al., 2010b; Kaufling et al., 2010; James et al., 2011; Lecca et al., 2011). These findings suggest that druginduced changes in the DA circuit might be, at least in part, a consequence of adaptations taking place in structures upstream of the VTA, such as the LHb. However, the effects of drug exposure on synaptic transmission and plasticity in the LHb remain unknown.

Here, we used tract tracing in combination with ex vivo patch-clamp recordings to demonstrate that exposure to cocaine selectively potentiates AMPAR-mediated transmission in $\mathrm{LHb}$ neurons projecting to the RMTg $\left(\mathrm{LHb}^{\rightarrow \mathrm{RMTg}}\right)$, but not to the VTA $\left(\mathrm{LHb}^{\rightarrow \mathrm{VTA}}\right)$, via a postsynaptic increase in AMPAR content. Consequently, cocaine-evoked synaptic potentiation modifies the rules for long-term plasticity in LHb neurons.

\section{Materials and Methods}

Surgery. C57BL/6J mice (20-40 d old) of either sex were used in accordance with the guidelines of the French Agriculture and Forestry Ministry for handling animals. Animals were anesthetized with ketamine (90 $\mathrm{mg} / \mathrm{kg}$ )/xylazine $(15 \mathrm{mg} / \mathrm{kg}$ ) (Sigma-Aldrich) before bilateral injection with either Cholera-toxin-B-Alexa Fluor 488/594 or fluorobeads (Invitrogen, Lumafluor). The following coordinates were used (from bregma, in $\mathrm{mm}$ ): RMTg: anteroposterior (A-P), -2.9 ; mediolateral (M-L), \pm 0.5 ; dorsoventral (D-V), -4.3; VTA: A-P, -2.4 ; M-L, \pm 0.8 ; D-V, -4.4. Recovery was allowed for at least $3 \mathrm{~d}$.

Electrophysiology. Animals injected with saline or cocaine $(10 \mathrm{mg} / \mathrm{kg})$, once for 2 consecutive days, were anesthetized (ketamine/xylazine) $24 \mathrm{~h}$ after the last injection for preparation of LHb-containing brain slices. 
A
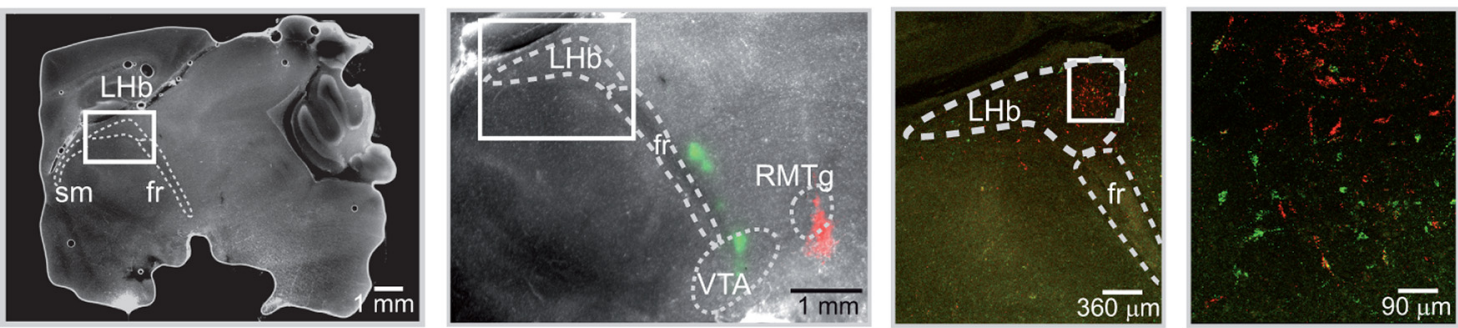

B

$\mathrm{LHb} \rightarrow \mathrm{RMTg}$

C

D
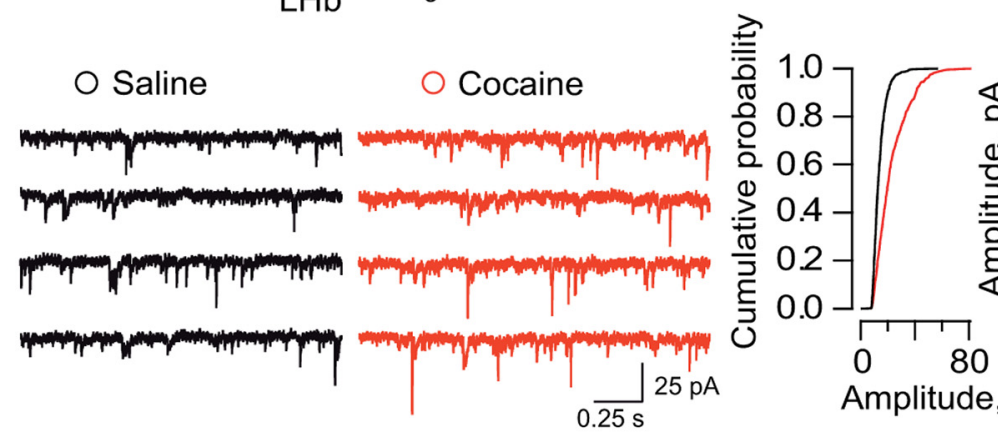

Amplitude, pA

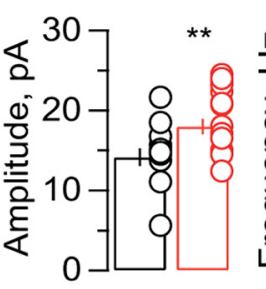

Sal Coc

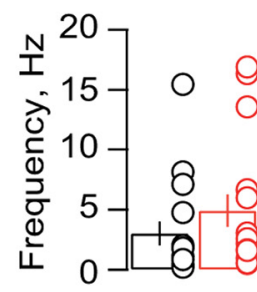

Sal Coc
E

Saline

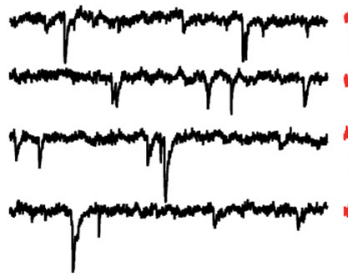

$\mathrm{LHb} \rightarrow \mathrm{VTA}$

Cocaine

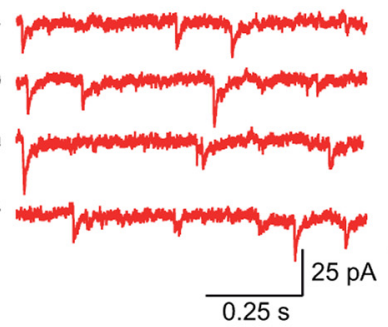

$\mathbf{F}$

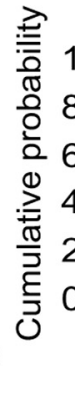

$\mathbf{G}$

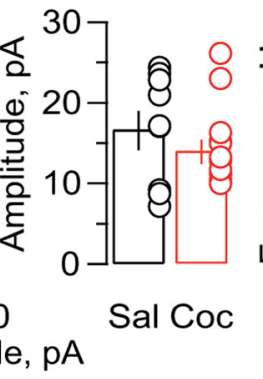

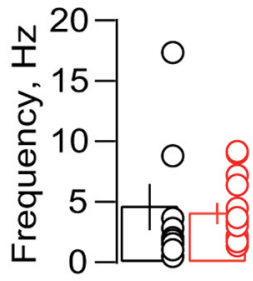

Sal Coc

Figure 1. Cocaine increases the efficacy of excitatory transmission onto LHb $\rightarrow$ RMTg neurons. $A, L$ LHb-containing sagittal sections illustrating the injections sites with retrobeads in the RMTg (red) and the VTA (green, left panels). Confocal images $(10 \times / 40 \times)$ illustrating retrogradely labeled LHb $\rightarrow$ RMTg and LHb $\rightarrow$ VTA neurons (right panels). sm, Stria medullaris; fr, fasciculus retroflexus. $\boldsymbol{B}$, Sample traces of mEPSCs obtained in LHb $\rightarrow{ }^{\mathrm{RMTg}}$ neurons in slices from saline-treated (black) and cocaine-treated (red) mice. $C$, Cumulative probability distributions (KS test, $\left.p<0.0001\right)$ for a

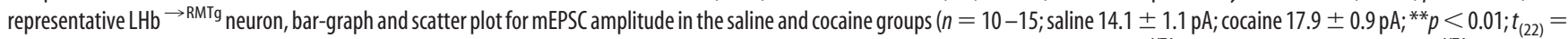
2.9). $\boldsymbol{D}$, Same as $\boldsymbol{C}$ for mEPSC frequency $\left(n=10-15\right.$; saline $3 \pm 0.9 \mathrm{~Hz} ;$ cocaine $\left.4.9 \pm 1.3 \mathrm{~Hz} ; p>0.05 ; t_{(22)}=0.9\right)$. $\boldsymbol{E}$, Same as $\boldsymbol{B}$ for $L \mathrm{Hb} \rightarrow \mathrm{VTA}$ neurons. $\boldsymbol{F}$, Same as $\boldsymbol{C}$ for $L \mathrm{Hb} \rightarrow \mathrm{VTA}$ neurons $(\mathrm{KS}$ test, $p>0.05)\left(n=9-14\right.$; saline $16.7 \pm 2.2 \mathrm{pA}$; cocaine $\left.14.08 \pm 1.3 \mathrm{pA} ; p>0.05 ; t_{(21)}=1.1\right)$. G, Same as $\boldsymbol{F}$ for mEPSC frequency $(n=9-14 ;$ saline $4.6 \pm 1.8 \mathrm{~Hz} ;$ cocaine $4.1 \pm 0.7 \mathrm{~Hz} ; p>$ $\left.0.05 ; t_{(21)}=0.3\right)$. Sal, Saline; Coc, cocaine.

Slicing was performed in bubbled ice-cold $95 \% \mathrm{O}_{2} / 5 \% \mathrm{CO}_{2}$-equilibrated solution containing the following (in $\mathrm{mM}$ ): choline $\mathrm{Cl} \mathrm{110}$; glucose 25; $\mathrm{NaHCO}_{3} 25 ; \mathrm{MgCl}_{2} 7$; ascorbic acid 11.6; $\mathrm{Na}^{+}$-pyruvate 3.1; $\mathrm{KCl}$ 2.5; $\mathrm{NaH}_{2} \mathrm{PO}_{4}$ 1.25; and $\mathrm{CaCl}_{2}$ 0.5. Sagittal slices $(250 \mu \mathrm{m})$ were stored at room temperature in $95 \% \mathrm{O}_{2} / 5 \% \mathrm{CO}_{2}$-equilibrated artificial CSF containing the following (in mM): $\mathrm{NaCl} 124 ; \mathrm{NaHCO}_{3} 26.2$; glucose $11 ; \mathrm{KCl}$ 2.5; $\mathrm{CaCl}_{2} 2.5 ; \mathrm{MgCl}_{2}$ 1.3; and $\mathrm{NaH}_{2} \mathrm{PO}_{4}$ 1. Recordings (flow rate of 2.5 $\mathrm{ml} / \mathrm{min}$ ) were made under an Olympus BX51 microscope at $32^{\circ} \mathrm{C}$. Currents were amplified, filtered at $5 \mathrm{kHz}$, and digitized at $20 \mathrm{kHz}$. Access resistance was monitored by a step of $-10 \mathrm{mV}(0.1 \mathrm{~Hz})$. Experiments were discarded if the access resistance increased $>20 \%$. Miniature EPSCs (mEPSCs) were recorded in voltage-clamp mode at $-60 \mathrm{mV}$ in the presence of bicuculline $(20 \mu \mathrm{M})$, TTX $(1 \mu \mathrm{M})$, and D,L $(-)$-2-amino-5phosphonovaleric acid (D,L-AP5; $100 \mu \mathrm{M})$. The internal solution contained the following (in mM): $\mathrm{CsCl} \mathrm{130;} \mathrm{NaCl} 4 ; \mathrm{MgCl}_{2}$ 2; EGTA 1.1; HEPES 5; $\mathrm{Na}_{2}$ ATP 2; $\mathrm{Na}^{+}$-creatine-phosphate 5; $\mathrm{Na}_{3} \mathrm{GTP} 0.6$; and spermine 0.1 was added when indicated. The liquid junction potential was $-3 \mathrm{mV}$. EPSCs were evoked through glass electrodes placed in the stria medullaris. AMPA/NMDA ratios of evoked EPSC were obtained by AMPA-EPSC $+40 \mathrm{mV} / \mathrm{NMDA}$-EPSCs at $+40 \mathrm{mV}$ as previously described (Ungless et al., 2001). The rectification index (RI) was calculated by the formula (EPSC -70/EPSC +40)/1.75 in the presence of D,L-AP5 and intracellular spermine. For long-term plasticity, the internal solution contained the following (in $\mathrm{mm}$ ): K-gluconate 140; $\mathrm{KCl} 5$; HEPES 10; EGTA 0.2; $\mathrm{MgCl}_{2} 2 ; \mathrm{Na}_{2} \mathrm{ATP} 4 ; \mathrm{Na}_{3} \mathrm{GTP} 0.3$; and creatine-phosphate 10 . Recordings were corrected for liquid junction potential $(12 \mathrm{mV})$. The induction protocol for long-term plasticity consisted of a $10 \mathrm{~Hz}$ stimulation (20 times each $5 \mathrm{~s})$ paired with somatic hyperpolarization $(-90$ $\mathrm{mV})$. Recordings were kept for at least 30-45 min.

For glutamate uncaging (4-methoxy-7-nitroindolinyl-caged L-glutamate $200 \mu \mathrm{M}$, Tocris-Cookson), a single-path photolysis head (Prairie Technologies) was connected to a solid-state laser $(405 \mathrm{~nm}$, duration $0.5 \mathrm{~ms}$, diameter 3-5 $\mu \mathrm{m}$ ). Photolysis was optimized by adjusting the laser beam position and power (3-10 mW) such that laser-evoked EPSCs [(L)-EPSCs] yielded amplitudes and kinetics similar to mEPSCs. AMPA/NMDA ratios of (L)-EPSCs were obtained by dividing the peak of AMPA-EPSC at $-60 \mathrm{mV}$ and the NMDA-EPSCs at $+40 \mathrm{mV}$ at $30 \mathrm{~ms}$ after onset as previously described (Mameli et al., 2011).

Nonstationary fluctuation analysis. A peak-scaled nonstationary fluctuation analysis (NSFA) was performed on mEPSCs (100-200) (Synaptosoft). mEPSCs were selected by: fast rise time alignment, stable baseline holding current, and the absence of spurious fluctuations during the 
A

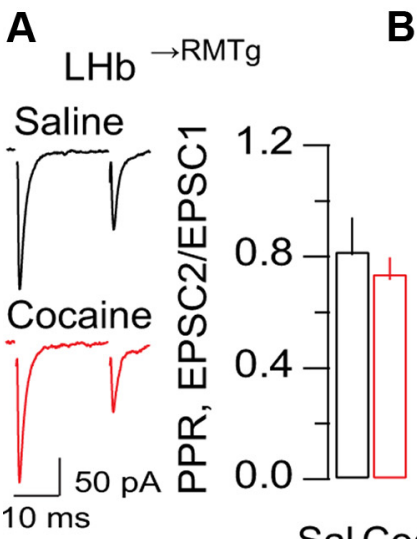

B
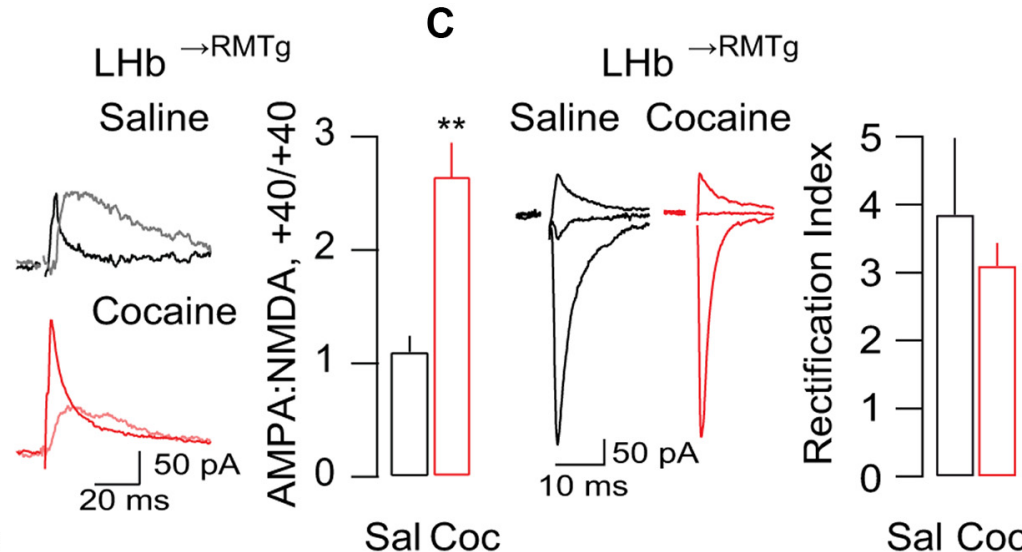

D $\quad$ LHb $\rightarrow$ RMTg

E

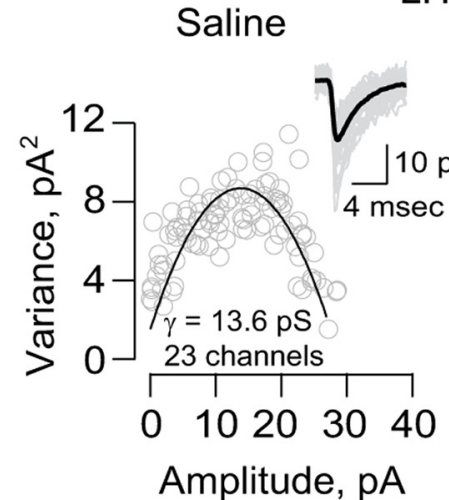

Cocaine
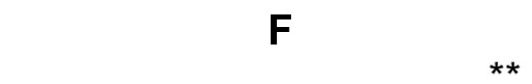

Sal Coc

Amplitude, pA

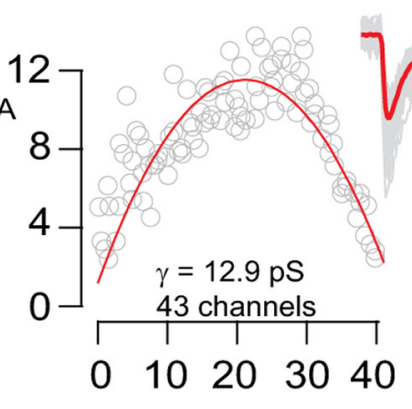

Amplitude, $\mathrm{pA}$

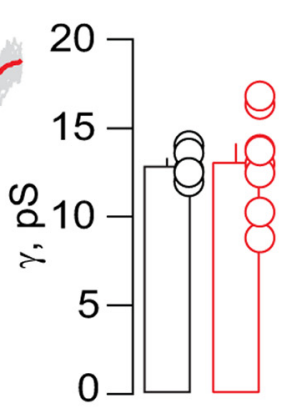

Sal Coc

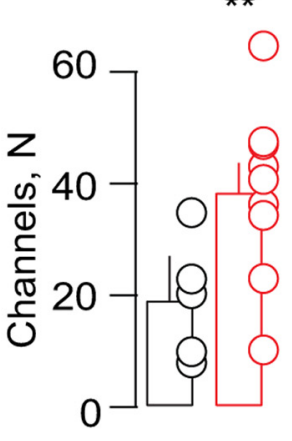

Sal Coc

Figure 2. Synaptic potentiation in $\mathrm{LHb} \rightarrow$ RMTg neurons requires a larger synaptic pool of AMPARs with no change in subunit composition. $A$, Averaged traces and bar-graph illustrating the PPR in the saline and cocaine groups (saline $0.82 \pm 0.1 ;$ cocaine $0.74 \pm 0.05 ; p>0.05 ; t_{(12)}=0.64 ; n=6-7$ ). B, Sample traces illustrating AMPA and NMDA-EPSCs recorded at $+40 \mathrm{mV}$ and AMPA/NMDA ratios in LHb $\rightarrow$ RMTg neurons in slices from saline (black) and cocaine (red) groups $\left(n=5-7\right.$; saline $1.1 \pm 0.1 ;$ cocaine $2.6 \pm 0.3 ;{ }^{* *} p<0.01 ; t_{(9)}=4.9$ ). C, Sample traces of AMPA-EPSCs recorded at $-70,0$, and $+40 \mathrm{mV}$ and RIs in LHb $\rightarrow$ RMTg neurons in slices from saline (black) and cocaine (red) groups $\left(n=6 ;\right.$ saline $3.85 \pm 1.12 ; c$ cocaine $3.1 \pm 0.3 ; p>0.05 ; t_{(15)}=$ 0.7). D. Example of peak-scaled NSFA of $L \mathrm{Hb} \rightarrow$ RMTg neurons in the saline- and cocaine-treated group. Insets, Overlay of 50 consecutive traces. $E, F$, Pooled data for conductance $(\gamma)$ and number of channels $(N)$ open at the peak $\left(n=5-8 ; \gamma\right.$ : saline $12.9 \pm 0.4 \mathrm{pS}$; cocaine $13.1 \pm 0.9 \mathrm{pS} ; p>0.05 ; t_{(11)}=0.1 ; N$ : saline $19.2 \pm 4.8 ;$ cocaine $\left.42 \pm 4.2 ;{ }^{* *} p<0.01 ; t_{(11)}=3.4\right)$. Sal, Saline; Coc, cocaine.

mEPSCs decay. The variance-amplitude relationship of mEPSC decay was plotted and fitted with the equation $\sigma^{2}=i I-I^{2} / N+\sigma_{b}^{2}$ (where $i$ is the mean single-channel AMPA current, $I$ is the mean current, $N$ is the number of channels activated at the peak, $N=$ mean amplitude/ $i$; and $\sigma_{b}{ }^{2}$ is the baseline variance). $i$ was estimated as the slope of the linear fit of the first portion of the parabola of the fitted mEPSC decay. The goodness-of-fit was assessed with a least-squares algorithm. The unitary current was converted in conductance based on the reversal potential of evoked EPSCs $(\sim 0 \mathrm{mV})$. Conductance and average EPSC amplitude, mean rise time, mean decay time, access resistance, or background noise variance had no correlation $(p>0.4)$ (Balland et al., 2006).

Analysis and drugs. All drugs were obtained from Abcam and Tocris Bioscience and dissolved in water, except for TTX (citric acid 1\%) and cocaine $\mathrm{HCl}(\mathrm{NaCl} 0.9 \%)$. On-line/off-line analyses were performed using IGOR-6 (Wavemetrics) and Prism (Graphpad). Compiled data are expressed as the mean \pm SEM. Significance was set at $p=0.05$ using Student's $t$ test and the Kolmogorov-Smirnov (KS) test.

\section{Results}

\section{Cocaine strengthens excitatory synaptic transmission in} the LHb

LHb neurons send axons to the RMTg and the VTA (BalcitaPedicino et al., 2011; Hong et al., 2011). To selectively identify $\mathrm{LHb} \rightarrow \mathrm{RMTg}$ and $\mathrm{LHb}^{\rightarrow \mathrm{VTA}}$ neurons, we stereotaxically injected green and red fluorobeads in the VTA and RMTg, re- spectively. As previously shown in rats (Li et al., 2011), $\mathrm{LHb}^{\rightarrow \mathrm{RMTg}}$ and $\mathrm{LHb} \rightarrow \mathrm{VTA}$ neuronal populations were segregated (Fig. 1A).

Addictive drugs modify excitatory synaptic transmission in the mesocorticolimbic system (Lüscher and Malenka, 2011). We examined whether cocaine affects excitatory synaptic transmission onto LHb neurons. Mice infused with retrograde tracers in the RMTg and/or VTA were injected with cocaine/ saline for 2 consecutive days, a paradigm leading to a progressive rise in locomotor activity (data not shown). We prepared LHb-containing slices $24 \mathrm{~h}$ after the last injection, and performed whole-cell recordings from retrogradely labeled $\mathrm{LHb}$ neurons. We first monitored quantal release by recording pharmacologically isolated AMPAR-mediated mEPSCs. In saline-treated animals, mEPSC amplitude and frequency in $\mathrm{LHb} \rightarrow \mathrm{RMTg}$ and $\mathrm{LHb}^{\rightarrow \mathrm{VTA}}$ neurons were comparable (Fig. $1 B-G$; amplitude, $t_{(17)}=1.3$; frequency, $\left.t_{(16)}=0.8 ; p>0.05\right)$. After cocaine, mEPSCs amplitude significantly increased in

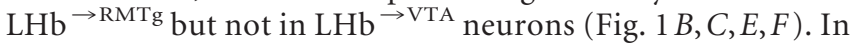
contrast, mEPSC frequency was not significantly affected regardless of the neuronal subpopulation (Fig. $1 B, D, E, G$ ). These findings suggest that cocaine exposure strengthens AMPARmediated synaptic transmission specifically in $\mathrm{LHb}^{\rightarrow \mathrm{RMTg}}$ neurons. 
A

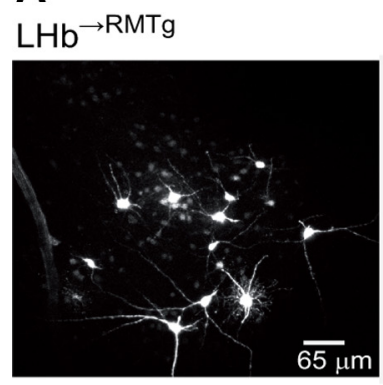

B

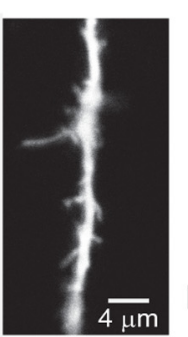

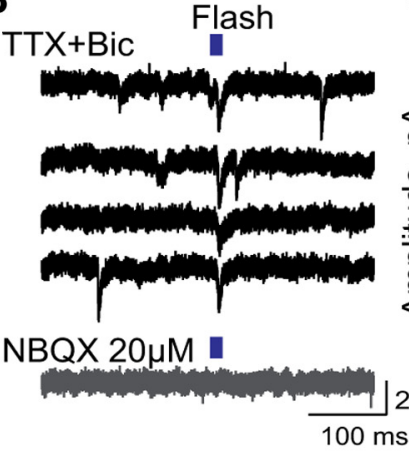

C

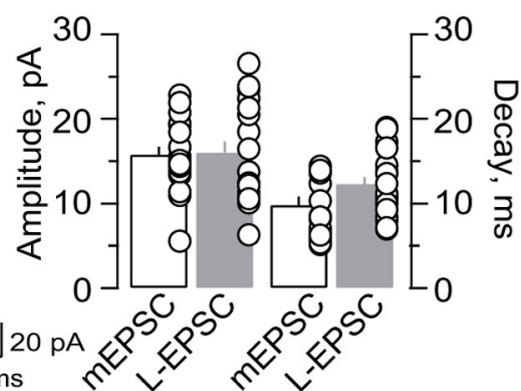

D

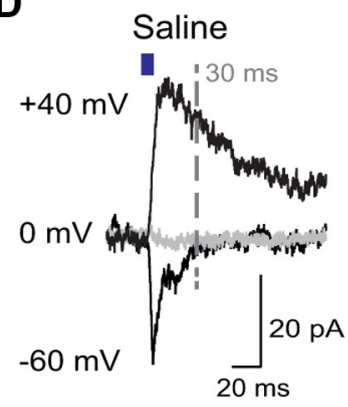

Cocaine

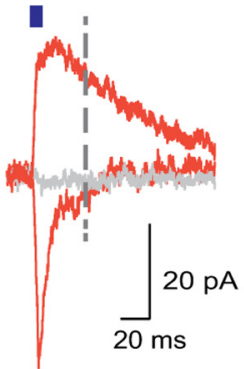

E

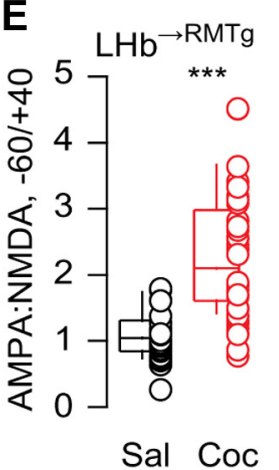

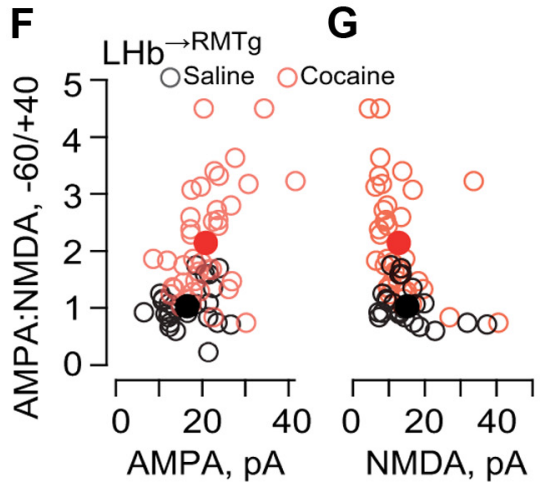

Figure 3. Cocaine-evoked plasticity in LHb $\rightarrow$ RMTg neurons is postsynaptic. $\boldsymbol{A}$, Confocal image of LHb neurons filled with Alexa Fluor Red-594 (left) and magnified dendrite (right). $\boldsymbol{B}$, Sample mEPSCs and (L)-EPSCs. Note blockade of all events with NBQX. C, Mean values and scatter plot for mEPSC and (L)-EPSC amplitudes and decays (90-37\%) in naive animals ( $n=20)$. $\boldsymbol{D}$, Averaged traces of (L)-EPSC at $-60,0$, and $+40 \mathrm{mV}$ in slices from saline and cocaine-treated animals. $E$, Box and scatter plot for the AMPA/NMDA ratios in the saline and cocaine groups ( $n=9-13$ cells and 24-39 synapses; $\left.{ }^{* * *} p<0.001 ; t_{(61)}=5.3\right)$. $\boldsymbol{F}, \mathbf{G},(\mathrm{L})$-AMPA-EPSC (L-AMPA) and L-NMDA-EPSC (L-NMDA) vs AMPA/NMDA ratio, scatter plot (empty circles) and mean values (full circles) (L-AMPA-EPSCs: saline $16.5 \pm 1.12 \mathrm{pA}$; cocaine $20.7 \pm 1 ;{ }^{* *} p<0.01 ; t_{(61)}=2.68 ;(\mathrm{L})$-NMDA-EPSCs: saline $15.1 \pm 1.6 \mathrm{pA}$; cocaine $\left.12.8 \pm 1.7 ; p>0.05 ; t_{(61)}=0.96\right)$. Sal, Saline; Coc, cocaine.

\section{Cocaine postsynaptically potentiates AMPAR-mediated transmission in $\mathrm{LHb} \rightarrow$ RMTg neurons}

We next examined the effect of cocaine on synaptically evoked EPSCs. Under our recording conditions, paired-pulse depression was detected in $\mathrm{LHb}^{\rightarrow \mathrm{RMTg}}$ neurons and the paired-pulse ratio (PPR) of AMPAR-EPSCs was not affected by cocaine exposure (Fig. 2A), indicating no alterations in presynaptic release. We next compared the ratio of AMPAR and NMDAR-EPSCs recorded at $+40 \mathrm{mV}$ (AMPA/NMDA). Cocaine exposure increased the AMPA/NMDA ratio in $\mathrm{LHb}^{\rightarrow \mathrm{RMTg}}$ neurons (Fig. $2 \mathrm{~B}$ ), indicating a postsynaptic strengthening of excitatory transmission. Next, we assessed the properties of AMPARs by recording EPSCs at $-70,0$, and $+40 \mathrm{mV}$, and calculating the $\mathrm{RI}$ in $\mathrm{LHb} \rightarrow \mathrm{RMTg}$ neurons. As shown in rats (Li et al., 2011), we also observed large RIs in mice, suggesting that AMPARs in LHb lack the GluA2 subunit (Fig. 2C). In the VTA, a single injection of cocaine leads to a change in AMPAR subunit composition from GluA2containing to GluA2-lacking (Bellone and Lüscher, 2006; Mameli et al., 2011). We found that the RIs in LHb $\rightarrow$ RMTg neurons from the saline and cocaine groups were not significantly different (Fig. 2C), indicating that cocaine treatment did not change AMPAR subunit composition.

To assess whether cocaine treatment affected AMPAR conductance or receptor number, we used peak-scaled NSFA of mEPSCs recorded from $\mathrm{LHb}^{\rightarrow \mathrm{RMTg}}$ neurons. The estimated $i$ value was not affected by cocaine treatment (Fig. $2 D, E$ ). On the other hand, the estimated number of channels opened at the peak was significantly larger ( $N$; Fig. $2 D, F)$. This suggests that cocaine induces an increase in the number of AMPARs without changing their biophysical properties or their subunit composition.
We next characterized the effect of cocaine exposure on AMPAR-mediated currents evoked by glutamate uncaging. We filled LHb $\rightarrow$ RMTg neurons with dye to visualize cell morphology and performed recordings in slices from saline- or cocainetreated animals using focalized photolysis of caged glutamate in dendrites (Fig. 3A). While amplitudes of laser-evoked EPSCs at $-60 \mathrm{mV}$ were comparable to those of mEPSCs, these events had slower decay, suggesting activation of synaptic as well as extrasynaptic glutamate receptors by glutamate uncaging (Fig. $3 B, C$ ). (L)-EPSCs were mediated by AMPARs, as they were blocked by NBQX (Fig. 3B). We then mapped synaptic hot spots by uncaging glutamate at $-60,0$, and $+40 \mathrm{mV}$. (L)-EPSCs at depolarized potentials had slower decay kinetics, indicating activation of both AMPARs and NMDARs (Fig. 3D). We found that AMPA/ NMDA ratios of laser-evoked currents (AMPA $-60 \mathrm{mV}$, peak/ $\mathrm{NMDA}_{+40 \mathrm{mV}}, 30 \mathrm{~ms}$ onset) in $\mathrm{LHb} \rightarrow \mathrm{RMTg}$ neurons of slices from cocaine-treated animals were significantly larger than those from saline-treated animals (Fig. $3 E$ ). Plotting the ratio as a function of the (L)-AMPA-EPSCs revealed that cocaine significantly shifts the distribution toward the top right (Fig. $3 F$ ), while no significant changes were detected for (L)-NMDA currents (Fig. 3G). These findings support that the increase in AMPAR-mediated component is largely responsible for the increase in AMPA/ NMDA ratio. Altogether, these results suggest that cocaine triggers a synaptic potentiation by enhancing the AMPAR content at excitatory synapses onto $\mathrm{LHb} \rightarrow \mathrm{RMTg}$ neurons.

\section{Cocaine drives metaplasticity in the LHb}

Hippocampal interneurons and DA neurons in the VTA undergo long-term synaptic changes through GluA2-lacking AMPAR- 
A
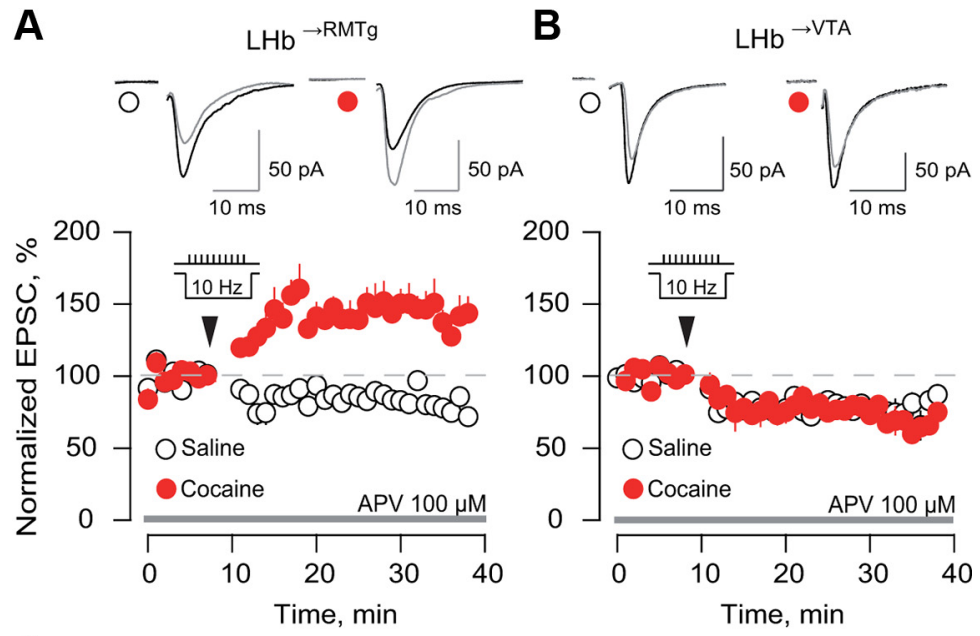

C
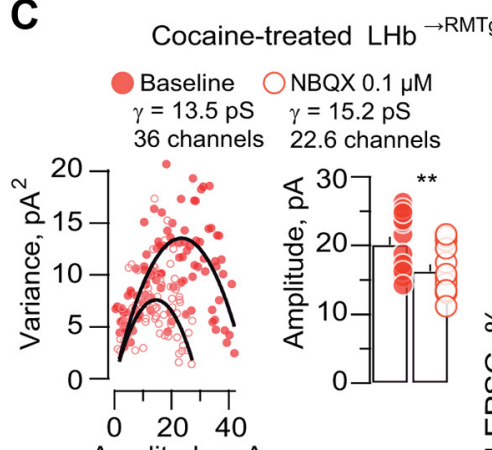

Amplitude, $\mathrm{pA}$
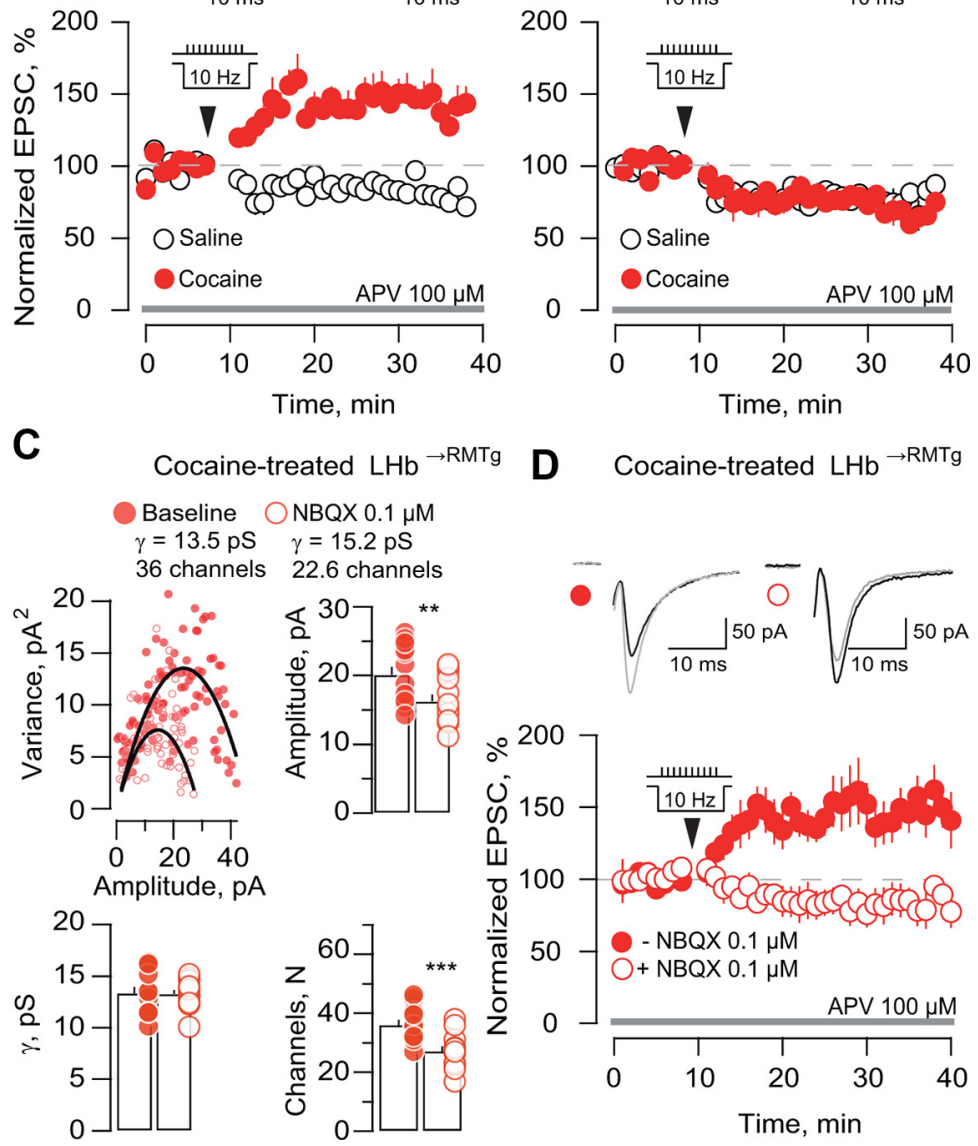

\section{D} Cocaine-treated $\mathrm{LHb} \rightarrow \mathrm{RMTg}$
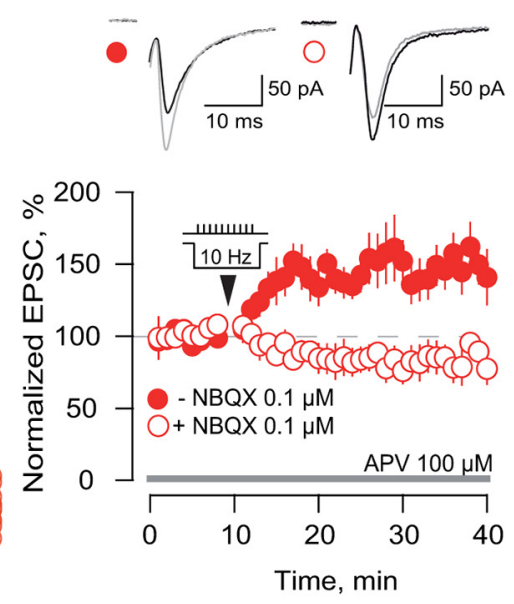

Figure 4. Cocaine evokes metaplasticity in $\mathrm{LHb} \rightarrow \mathrm{RMTg}$ neurons. $\boldsymbol{A}$, Averaged traces and amplitudes versus time plot of AMPAREPSCs in LHb $\rightarrow$ RMTg neurons before and after conditioning protocol (arrow) in slices of saline and cocaine groups ( $n=6-8$; saline $86.6 \pm 3.7 \%$; cocaine $\left.145.2 \pm 11.8 \% ; p<0.01 ; t_{(12)}=4.1\right)$. $\boldsymbol{B}$, Same as $\boldsymbol{A}$ but for LHb $\rightarrow$ VTA neurons $(n=5-6$; saline $77.9 \pm$ $4.1 \%$; cocaine $\left.78.2 \pm 2.5 \% ; p>0.05 ; t_{(10)}=0.04\right)$. C, Example of peak-scaled NSFA of mEPSCs of an LHb $\rightarrow$ RMTg neuron before and after bath application of $0.1 \mu \mathrm{M}$ NBQX in the cocaine group. Pooled data for amplitudes, conductances $(\gamma)$, and number of channels $(N)$ open at the peak of the response $\left(n=13\right.$; amplitude: baseline $20.1 \pm 1.2 \mathrm{pA} ; \mathrm{NBQX} 16.2 \pm 1 \mathrm{pA} ;{ }^{* *} p<0.01 ; t_{(12)}=$ 6.7; $\gamma$ : baseline $13.3 \pm 0.6 \mathrm{pS}, \mathrm{NBQX} 13.3 \pm 0.4 \mathrm{pS} ; p>0.5 ; t_{(12)}=0.1 ; \mathrm{N}$ : baseline $36.1 \pm 1.7 ; \mathrm{NBQX} 27.2 \pm 1.6 ;{ }^{* * *} p<0.001$; $\left.t_{(12)}=6.6\right) . D$, Averaged sample traces and amplitude versus time plot of AMPAR-EPSCs from LHb $\rightarrow$ RMTg neurons before and after the conditioning protocol in slices from cocaine-exposed animals in the presence or absence of NBQX $0.1 \mu \mathrm{M}(n=7$; control $\left.149.3 \pm 15.5 \% ; \mathrm{NBQX} 80.42 \pm 9.9 \% ;{ }^{* *} p<0.01 ; t_{(12)}=3.7\right)$

mediated calcium influx (Lamsa et al., 2007; Mameli et al., 2011). Given that cocaine increases the levels of AMPARs in LHb $\rightarrow$ RMTg neurons, we asked whether cocaine experience altered long-term synaptic plasticity rules. We tested whether synaptic activity modulated AMPAR-mediated transmission onto $\mathrm{LHb} \rightarrow$ RMTg and LHb $\rightarrow$ VTA neurons. GluA2-lacking AMPARs conduct more efficiently at hyperpolarized potentials, therefore we used a synaptic protocol pairing presynaptic activity with postsynaptic hyperpolarization in presence of the NMDAR blocker APV (Fig. $4 A, B)$ (Lamsa et al., 2007).

In saline-treated mice, we found that a $10 \mathrm{~Hz}$ stimulation protocol induced a synaptic depression in both $\mathrm{LHb} \rightarrow \mathrm{RMTg}$ and $\mathrm{LHb}^{\rightarrow \text { VTA }}$ neurons (Fig. $4 A, B$ ). In contrast, after cocaine treatment, a robust LTP was observed specifically in $\mathrm{LHb}^{\rightarrow \mathrm{RMTg}}$ neurons (Fig. $4 A, B$ ). To causally link the cocaine-evoked increase in quantal size $(\sim 20 \%)$ and the metaplasticity in $\mathrm{LHb} \rightarrow$ RMTg neu- rons, we used a submaximal concentration of NBQX $(0.1 \mu \mathrm{M})$ that decreased mEPSC amplitude by $\sim 20 \%$, mimicking AMPAR synaptic transmission in the control group (Fig. 4C). Peak-scaled NSFA of mEPSCs revealed that NBQX $0.1 \mu \mathrm{M}$ decreased the number of channels opened at the peak without altering single-channel conductance (Fig. 4C). We then tested whether NBQX $0.1 \mu \mathrm{M}$ could restore the normal pattern of synaptic plasticity in $\mathrm{LHb}^{\rightarrow \mathrm{RMTg}}$ neurons. We found that the $10 \mathrm{~Hz}$ protocol in the presence of $0.1 \mu \mathrm{M}$ NBQX in slices from cocaine-treated mice produced a synaptic depression similar to that observed in slices from saline-treated mice (Fig. 4D). The cocaine-mediated LTP was intact in interleaved slices in the absence of NBQX. Hence, cocaine-evoked potentiation enables metaplasticity in LHb $\rightarrow$ RMTg neurons.

\section{Discussion}

Using whole-cell recordings in conjunction with retrograde labeling techniques, we find that cocaine experience increases AMPAR-mediated transmission onto $\mathrm{LHb} \rightarrow \mathrm{RMTg}$ neurons by increasing the number of AMPARs at the postsynaptic level. These results indicate that cocaine profoundly alters the efficacy of excitatory transmission in LHb in a projection-specific fashion. This condition further allows presynaptic activity to trigger a form of LTP, absent in control animals, ultimately diverting the induction rules for long-term plasticity.

Our data suggest that the subunit composition of the synaptic AMPARs in $\mathrm{LHb}^{\rightarrow \mathrm{RMTg}}$ neurons was not significantly changed by cocaine treatment. Recent electrophysiological evidence in rats indicates the presence of GluA2-lacking AMPARs in these neurons (Li et al., 2011). In agreement with this study, we found rectifying EPSCs in mouse slices, consistent with the expression of GluA2-lacking AMPARs. However, a residual current at positive potentials was still detected, suggesting that excitatory synapses onto LHb neurons likely contain GluA2-lacking and GluA2-containing AMPARs. Importantly, cocaine did not change the RI of $\mathrm{LHb}^{\rightarrow \mathrm{RMTg}}$ neurons, indicating no alterations in AMPAR subunit composition. This differs from drug-evoked synaptic adaptations in the VTA or ventral striatum, where acute or chronic cocaine promotes the insertion of GluA2lacking AMPARs at synapses initially devoid of these receptors (Bellone and Lüscher, 2006; Conrad et al., 2008).

NSFA provides further insight into the cellular mechanism behind cocaine potentiation, revealing a larger pool of AMPARs. Consistently, the uncaging experiments suggest that larger AMPAEPSCs correlate with AMPA/NMDA ratios supporting the idea that synaptic strengthening relies, at least in part, on a higher number of postsynaptic AMPARs. 
Abraham and Bear (1996) defined metaplasticity as the ability of presynaptic activity to induce subsequent synaptic plasticity as a consequence of synaptic or cellular adaptations (Abraham and Bear, 1996). We demonstrate that cocaine-evoked synaptic potentiation of $\mathrm{LHb}^{\rightarrow \mathrm{RMTg}}$ neurons facilitates the induction of a form of metaplasticity absent in control conditions.

To demonstrate a causal relationship between cocaine-evoked synaptic strengthening and metaplasticity, we used low concentrations of NBQX to compensate for the cocaine-evoked synaptic potentiation. In cocaine-exposed mice, resetting synaptic transmission by reducing postsynaptic AMPAR availability is sufficient to restore the basal long-term synaptic plasticity. This evidence, together with larger quantal size, the increase in AMPA/ NMDA ratios, and the results from NSFA after cocaine, suggest the presence of a larger fraction of AMPARs, including some lacking GluA2. This may provide a higher synaptic calcium source responsible for the induction of cocaine-induced metaplasticity onto $\mathrm{LHb} \rightarrow{ }^{\mathrm{RMTg}}$ neurons.

Future studies are nevertheless required to understand the cascade of events leading to this cocaine-evoked synaptic potentiation in LHb. In vivo evidence demonstrates that DA neuron activity and $\mathrm{DA}$ receptor activation modulate the firing of LHb neurons. This implies a potential role of DA signaling in triggering cocaine-evoked adaptations in the LHb (Kowski et al., 2009; Shen et al., 2012). In the VTA, cocaine triggers synaptic plasticity via its action on the DA transporter (Brown et al., 2010a). Therefore, cocaine may lead to a surge in DA in LHb, which may account for the cellular mechanism triggering the synaptic potentiation onto LHb neurons.

Anatomical studies suggest that the LHb innervation onto RMTg is the principal candidate for the net inhibition of DA neurons (Balcita-Pedicino et al., 2011; Stamatakis and Stuber, 2012). Consistently with this view, we show that cocaine-evoked synaptic potentiation is selective for $\mathrm{LHb}^{\rightarrow \mathrm{RMTg}}$ neurons. From a neural circuit standpoint, the cocaine-evoked potentiation in $\mathrm{LHb}^{\rightarrow \mathrm{RMTg}}$ neurons may result in a stronger inhibition of DA neurons via RMTg GABA neurons, in line with recent proposed models in which drugs increase LHb firing, adaptively dampening DA release (Baldwin et al., 2011). In this context, the modulation of the LHb-to-RMTg pathway as well of GABA inputs impinging on DA neurons is crucial for the processing of rewarding and aversive stimuli (Stamatakis and Stuber, 2012; Tan et al., 2012). Future studies are required to better probe how cocaine, reward, or aversive experiences alter specific populations of DA neurons via the LHb-RMTg-VTA path (Lammel et al., 2011).

In conclusion, we provide evidence for a synaptic potentiation in the LHb that may represent a key cellular mechanism engaged in the formation of drug-associated memories. Cellular adaptations in LHb may not only contribute to the early phases of drug exposure as reported here, but also in shaping drug-seeking behaviors (Brown et al., 2010b; James et al., 2011). We propose that cocaine may lead to behavioral adaptations by modifying synaptic efficacy and the rules for long-term plasticity in LHb, participating in the dysregulation of the DA system.

\section{References}

Abraham WC, Bear MF (1996) Metaplasticity: the plasticity of synaptic plasticity. Trends Neurosci 19:126-130.

Balcita-Pedicino JJ, Omelchenko N, Bell R, Sesack SR (2011) The inhibitory influence of the lateral habenula on midbrain dopamine cells: ultrastructural evidence for indirect mediation via the rostromedial mesopontine tegmental nucleus. J Comp Neurol 519:1143-1164.

Baldwin PR, Alanis R, Salas R (2011) The role of the habenula in nicotine addiction. J Addict Res Ther S1:002.

Balland B, Lachamp P, Strube C, Kessler JP, Tell F (2006) Glutamatergic synapses in the rat nucleus tractus solitarii develop by direct insertion of calcium-impermeable AMPA receptors and without activation of NMDA receptors. J Physiol 574:245-261.

Bellone C, Lüscher C (2006) Cocaine triggered AMPA receptor redistribution is reversed in vivo by mGluR-dependent long-term depression. Nat Neurosci 9:636-641.

Brown MT, Bellone C, Mameli M, Labouèbe G, Bocklisch C, Balland B, Dahan L, Luján R, Deisseroth K, Lüscher C (2010a) Drug-driven AMPA receptor redistribution mimicked by selective dopamine neuron stimulation. PLoS One 5:e15870.

Brown RM, Short JL, Lawrence AJ (2010b) Identification of brain nuclei implicated in cocaine-primed reinstatement of conditioned place preference: a behaviour dissociable from sensitization. PLoS One 5:e15889.

Conrad KL, Tseng KY, Uejima JL, Reimers JM, Heng LJ, Shaham Y, Marinelli M, Wolf ME (2008) Formation of accumbens GluR2-lacking AMPA receptors mediates incubation of cocaine craving. Nature 454:118-121.

Hikosaka O (2010) The habenula: from stress evasion to value-based decision-making. Nat Rev Neurosci 11:503-513.

Hong S, Jhou TC, Smith M, Saleem KS, Hikosaka O (2011) Negative reward signals from the lateral habenula to dopamine neurons are mediated by rostromedial tegmental nucleus in primates. J Neurosci 31:11457-11471.

James MH, Charnley JL, Flynn JR, Smith DW, Dayas CV (2011) Propensity to "relapse" following exposure to cocaine cues is associated with the recruitment of specific thalamic and epithalamic nuclei. Neuroscience 199:235-242.

Jhou TC, Geisler S, Marinelli M, Degarmo BA, Zahm DS (2009) The mesopontine rostromedial tegmental nucleus: a structure targeted by the lateral habenula that projects to the ventral tegmental area of Tsai and substantia nigra compacta. J Comp Neurol 513:566-596.

Kaufling J, Veinante P, Pawlowski SA, Freund-Mercier MJ, Barrot M (2009) Afferents to the GABAergic tail of the ventral tegmental area in the rat. J Comp Neurol 513:597-621.

Kaufling J, Waltisperger E, Bourdy R, Valera A, Veinante P, Freund-Mercier MJ, Barrot M (2010) Pharmacological recruitment of the GABAergic tail of the ventral tegmental area by acute drug exposure. Br J Pharmacol 161:1677-1691.

Kowski AB, Veh RW, Weiss T (2009) Dopaminergic activation excites rat lateral habenular neurons in vivo. Neuroscience 161:1154-1165.

Lammel S, Ion DI, Roeper J, Malenka RC (2011) Projection-specific modulation of dopamine neuron synapses by aversive and rewarding stimuli. Neuron 70:855-862.

Lamsa KP, Heeroma JH, Somogyi P, Rusakov DA, Kullmann DM (2007) Anti-Hebbian long-term potentiation in the hippocampal feedback inhibitory circuit. Science 315:1262-1266.

Lecca S, Melis M, Luchicchi A, Ennas MG, Castelli MP, Muntoni AL, Pistis M (2011) Effects of drugs of abuse on putative rostromedial tegmental neurons, inhibitory afferents to midbrain dopamine cells. Neuropsychopharmacology 36:589-602.

Li B, Piriz J, Mirrione M, Chung C, Proulx CD, Schulz D, Henn F, Malinow R (2011) Synaptic potentiation onto habenula neurons in the learned helplessness model of depression. Nature 470:535-539.

Lüscher C, Malenka RC (2011) Drug-evoked synaptic plasticity in addiction: from molecular changes to circuit remodeling. Neuron 69:650-663.

Mameli M, Bellone C, Brown MT, Lüscher C (2011) Cocaine inverts rules for synaptic plasticity of glutamate transmission in the ventral tegmental area. Nat Neurosci 14:414-416.

Matsumoto M, Hikosaka O (2007) Lateral habenula as a source of negative reward signals in dopamine neurons. Nature 447:1111-1115.

Shen X, Ruan X, Zhao H (2012) Stimulation of midbrain dopaminergic structures modifies firing rates of rat lateral habenula neurons. PLoS One 7:e34323.

Stamatakis AM, Stuber GD (2012) Activation of lateral habenula inputs to the ventral midbrain promotes behavioral avoidance. Nat Neurosci 15:1105-1107.

Tan KR, Yvon C, Turiault M, Mirzabekov JJ, Doehner J, Labouèbe G, Deisseroth K, Tye KM, Lüscher C (2012) GABA neurons of the VTA drive conditioned place aversion. Neuron 73:1173-1183.

Ungless MA, Whistler JL, Malenka RC, Bonci A (2001) Single cocaine exposure in vivo induces long-term potentiation in dopamine neurons. Nature 411:583-587.

Wise RA (2004) Dopamine, learning and motivation. Nat Rev Neurosci 5:483-494. 